\title{
Calycosin may Alleviate Ang II-Induced Pro-proliferative Effects on Glomerular Mesangial Cells via Partially Inhibiting Autophagy and ERK Signaling Pathway
}

\author{
Xiaohuan Ding, ${ }^{a}$ Jing Lv, ${ }^{b}$ Jia Luan, ${ }^{a}$ and Jun Zhang*,c \\ ${ }^{a}$ Department of Integrated Traditional and Western Medicine, Liaoning University of Traditional Chinese Medicine; \\ Shenyang 110000, China: ${ }^{b}$ Department of Nephrology, Affiliated Hospital of Liaoning University of Traditional \\ Chinese Medicine; Shenyang 110000, China: and ${ }^{c}$ Department of Pediatrics, Affiliated Hospital of Liaoning \\ University of Traditional Chinese Medicine; Shenyang 110000, China. \\ Received June 23, 2020; accepted September 2, 2020; advance publication released online October 1, 2020
}

\begin{abstract}
Over-expression of angiotensin II (Ang II) is an important reason for the development of chronic kidney disease. Calycosin is the active component of traditional Chinese medicine astragali radix. The present work aims to explore whether calycosin could affect the growth and apoptosis ability of the Ang II treated glomerular mesangial cells and the underlying mechanism. Human glomerular mesangial cells (GMCs) were cultured and treated by Ang II and $0,0.1$, 1 , or $10 \mu \mathrm{M}$ calycosin, and the viability and proliferation of the cells were determined by methyl thiazolyl tetrazolium (MTT) and 5-ethynil-2'-deoxyuridine (EdU) staining; moreover, the apoptosis of the cells was examined by flow cytometry assay; furthermore, the expression levels of extracellular signal-regulated kinase (ERK), p-ERK, anti-apoptotic factor Bcl-2, as well as pro-apoptotic factor Bax have been examined by Western blot (WB) methods; finally, the expression of autophagic markers in each group was examined by WB and immunocytochemistry methods. We found that Ang II increased viability and proliferation, meanwhile inhibited apoptosis of the GMCs; furthermore, 1 and $10 \mu M$ calycosin significantly inhibited the growth and promoted the apoptosis of the GMCs treated by Ang II; moreover, calycosin also inhibited ERK signaling in mesangial cells activated by Ang II treatment; Finally, calycosin could inhibit Ang II induced autophagy of GMCs in a dose-dependent manner. In conclusion, calycosin may alleviate Ang II-induced pro-proliferative and anti-apoptotic effects on glomerular mesangial cells at least partially via inhibiting autophagy and ERK signaling pathway, suggesting that calycosin may function as a potential alternative medication for the management of chronic kidney diseases.
\end{abstract}

Key words calycosin; glomerular mesangial cell; proliferation; apoptosis; extracellular regulated protein kinase; autophagy

\section{INTRODUCTION}

In recent years, it has been widely accepted that the incidence of tubule interstitial fibrosis (TIF) was closely related to the incidence of chronic kidney disease (for example, diabetic nephropathy) which consequentially leads to renal failure. ${ }^{1,2)}$ Glomerular mesangial cells (GMCs) are groups of cells that function as key regulators in the renal system, and over-proliferation of GMCs was also considered as an important reason for the incidence and development of TIF. ${ }^{3-5)}$ Nevertheless, at the current stage, the roles of GMCs in the pathogenesis of TIF as well as the possible mechanism has not yet been fully elucidated.

Angiotensin II (Ang II) was a hormone that plays key roles in regulating the function of the renin-angiotensin system. ${ }^{6}$ Some recent studies have suggested the important regulatory functions of Ang II in renal system..$^{7-9)}$ Ang II was reported to participate in different biological processes, for example, cell growth, fibrosis, and immune reactions. Increased expression of Ang II can lead to increased growth ability and decreased apoptosis of the glomerular mesangial cells, which was considered as an important reason for the incidence and progress of TIF.,10)

With the development of medical science and pharmaceutical science, the therapeutic functions of extractives that derived from the natural plants ${ }^{11,12}$ have been investigated in many previous studies. Calycosin is the active component of traditional Chinese medicine Astragali Radix, and as many other natural extractives, it can be widely found and isolated from many kinds of plants, vegetables, and fruits. ${ }^{13-15)}$ Calycosin have exerted anti-oxidant, anti-fibrotic, anti-bacterial as well as anti-inflammatory activities. ${ }^{16,17)}$ In the field of renal diseases, the therapeutic effect of calycosin has also been reported. ${ }^{18-20)}$ The current work focused on the effects of calycosin on the growth, apoptosis, and autophagy of Ang II treated GMCs and the possible mechanism.

\section{MATERIALS AND METHODS}

Cell Culture and Treatment To determine the effects of calycosin on human glomerular mesangial cells (purchased from ATCC, VA, U.S.A.), GMCs were cultured and maintained in Dulbecco's modified Eagle's medium (DMEM) culture medium (Gibco, CA, U.S.A.) containing fetal bovine serum $(10 \%$, Gibco), penicillin and streptomycin $(100 \mathrm{U} / \mathrm{mL})$ at $37^{\circ} \mathrm{C}$ in an incubator with $5 \% \mathrm{CO}_{2}$. The calycosin groups were treated with the medium containing $0.1,1$, or $10 \mu \mathrm{M}$ calycosin (Sigma, St. Louis, MO, U.S.A.), respectively.

Western Blot GMCs were harvested and then lysed with the radioimmunoprecipitation (RIPA) methods (the kit was purchased from Beyotime, Haimen, China). Then the 
A
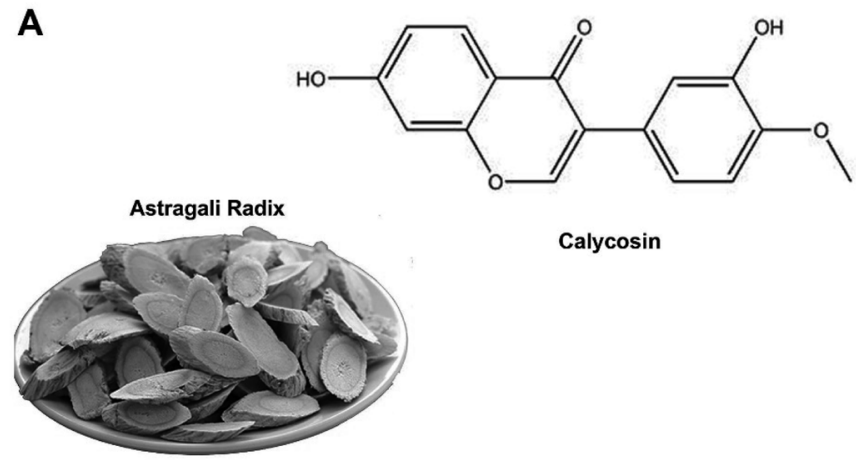

Calycosin

B

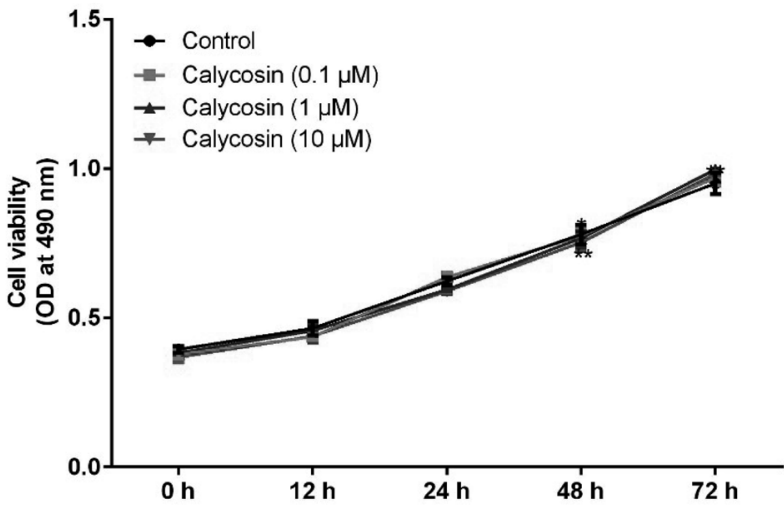

C

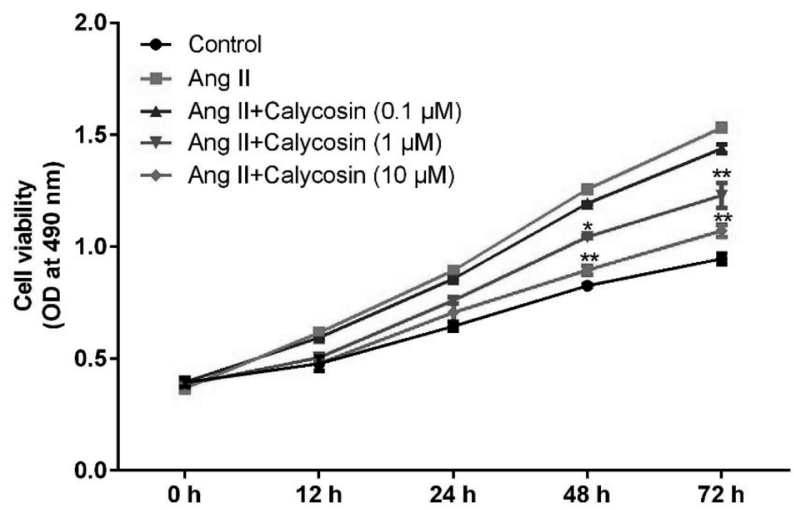

Fig. 1. A. Images of Astragali Radix and Calycosin: B. Effect of Calycosin on the Viability of Glomerular Mesangial Cells by MTT Assay: C. Effect of Calycosin on the Viability of Glomerular Mesangial Cells Treated by Ang II in Vitro by MTT Assay

$* p<0.05$ vs. Ang II, $* * p<0.01 v s$. Ang II.

proteins were separated by performing sodium dodecyl sulfate (SDS)-polyacrylamide gel electrophoresis. After the gel electrophoresis step, the separated proteins have been then transferred onto the polyvinylidene fluoride membrane. After the trans-membrane step, the membranes have been blocked by skimmed milk $(5 \%)$, incubated by different primary antibodies (including anti-extracellular signal-regulated kinase (ERK)1/2, anti-p-ERK1/2, anti-Bcl-2, anti-Bax, anti-Beclin-1 and anti-P62, and anti-glyceraldehyde-3-phosphate dehydrogenase (GAPDH), Abcam, Cambridge, MA, U.S.A.) overnight at $4{ }^{\circ} \mathrm{C}$. On day 2 , the membranes have been washed and the secondary antibodies (Beyotime) were added to the membrane
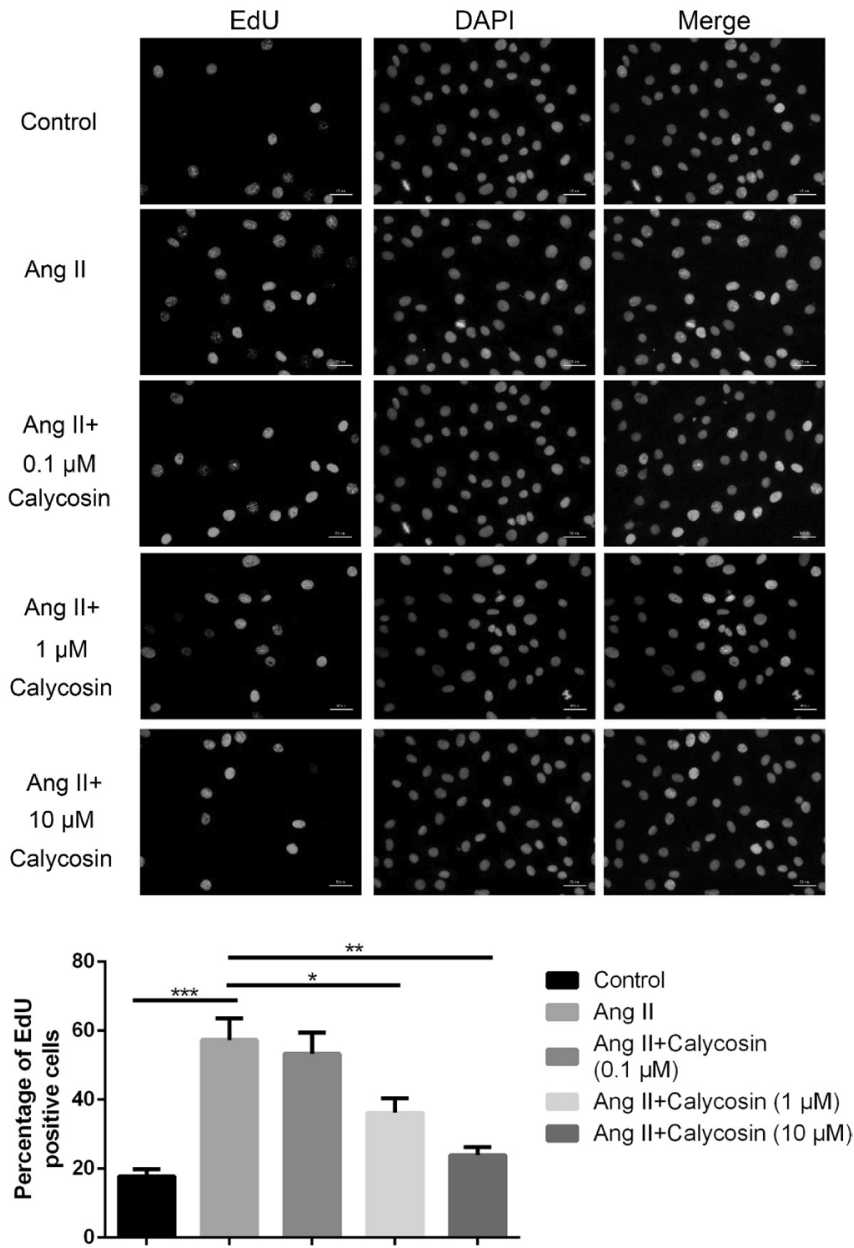

Fig. 2. Effect of Calycosin on the Viability of Glomerular Mesangial Cells Treated by Ang II in Vitro by EdU Staining

$* p<0.05,{ }^{* *} p<0.01, * * * p<0.001$. Scale bar $50 \mu \mathrm{m}$.

for $45 \mathrm{~min}$, and then washed and treated by the BeyoECL Plus kit for visualizing (Beyotime). The protein bands were imaged by the ChemiDoc ${ }^{\mathrm{TM}} \mathrm{XRS}^{+}$system (Bio-rad), and GAPDH has been applied for normalization.

Methyl Thiazolyl Tetrazolium (MTT) Assay Viability of GMCs has been evaluated by MTT assay. GMCs of different treatments were seeded onto 96-well plates, and $10 \mu \mathrm{L}$ MTT solution has been added to every well. After that, cell culture plates were incubated at $37^{\circ} \mathrm{C}$ for $4 \mathrm{~h}$. Then microplate reader (Thermo Fisher Scientific, MA, U.S.A.) has been used to measure the absorbance value $(490 \mathrm{~nm})$ to determine the viability of the cells.

5-Ethynil-2'-deoxyuridine (EdU) Labeling Cells were stained with EdU $(20 \mu \mathrm{M})$ from the Click-iT EdU Alexa Fluor 488 Imaging Kit (Beyotime, Shanghai, China) $48 \mathrm{~h}$ after treatment following the information of the manufacturer. After that, GMCs have been fixed by PFA (4\%), and the nuclei were stained by 4'-6-diamidino-2-phenylindole (DAPI). Cells were then imaged by a confocal microscope (Leica), and the data were analyzed by ImageJ software (NIH).

Cell Apoptosis Analysis GMCs were treated by the propidium iodide (PI)/Annexin V apoptosis kit (Beyotime) $48 \mathrm{~h}$ after different treatment. The apoptosis rate of cells with different treatments was examined by using the FACSVerse flow cytometer (BD Biosciences, NJ, U.S.A.) according to the 
instruction of the manufacturer.

Immunocytochemistry To evaluate the expressions of LC3 in GMCs of different treatments, immunocytochemistry methods have been performed. Briefly, cells were fixed by 4\% paraformaldehyde and incubated with anti-LC3 antibodies (Abcam) at room temperature for $30 \mathrm{~min}$, incubated by Alexa Fluor 488-conjugated secondary antibodies (Abcam) and then imaged by a fluorescent microscope.

Statistics All statistical analysis has been conducted by GraphPad Prism 7.0 software (GraphPad, CA, U.S.A.). Data have been shown as mean value \pm the standard deviation, oneway ANOVA method has been applied for the comparison of values among different groups. The $p$-value less than 0.05 has been considered as statistically significant.

\section{RESULTS}

Calycosin Can Inhibit the Viability of GMCs Treated by Ang II in Vitro Figure 1A shows the images of Astragali Radix and calycosin. First, GMCs were treated by $0,0.1,1$, or $10 \mu \mathrm{M}$ calycosin and the viability of the cells were examined using MTT method. We found that calycosin did not significantly affected the viability of GMCs (Fig. 1B, $p>0.05$ ); moreover, GMCs were treated by Ang II combined with $0,0.1$, 1 , or $10 \mu \mathrm{M}$ calycosin, and cell viability has been detected. We
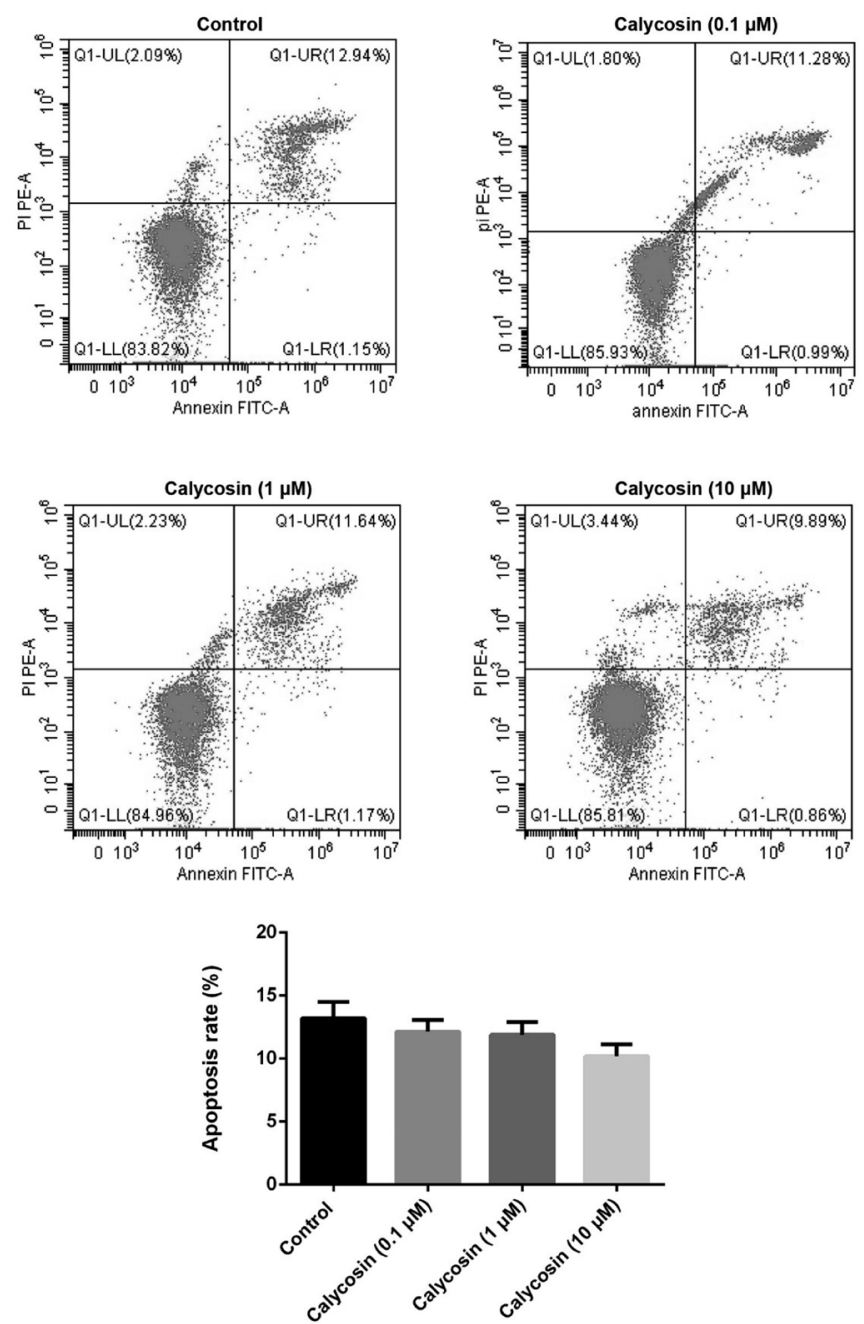

Fig. 3. Effect of Calycosin on the Apoptosis of Glomerular Mesangial Cells in Vitro by Flow Cytometry Assay found that Ang II treatment induced a dramatic increase in the viability of glomerular mesangial cells (Fig. 1B), while 1 and $10 \mu \mathrm{M}$ calycosin treatment inhibited the viability of GMCs treated by Ang II in a dose-as well as time-dependent manner. Meanwhile, $0.1 \mu \mathrm{M}$ calycosin has not shown inhibitory effects on the GMCs treated by Ang II $(p>0.05)$.

Calycosin Can Inhibit the Proliferation of GMCs Treated by Ang II in Vitro Moreover, the proliferation of the cells with different concentrations of calycosin was evaluated by EdU staining at $48 \mathrm{~h}$. As shown in Fig. 2, we found that Ang II promoted GMCs proliferation at $48 \mathrm{~h}$, and both 1 and $10 \mu \mathrm{M}$ calycosin significantly inhibits proliferation ability of GMCs treated by Ang II $(p<0.05)$. Meanwhile, the treatment of $0.1 \mu \mathrm{M}$ calycosin did not affect the proliferation ability of GMCs treated by Ang II (Fig. 2, $p>0.05$ ).

Calycosin Can Promote Apoptosis of GMCs Treated by Ang II Furthermore, the apoptosis of GMCs with different treatments were examined by flow cytometry assay. As shown in Fig. 3, after $48 \mathrm{~h}$ treatment, 0.1 , 1 , or $10 \mu \mathrm{M}$ calycosin did not significantly affected the apoptosis of GMCs (Fig. 3, $p>0.05$ ); on their other hand, Ang II markedly inhibited the apoptosis of glomerular mesangial cells, while 1 and $10 \mu \mathrm{M}$ calycosin markedly increased apoptosis of GMCs treated by Ang II (Fig. 4, $p<0.05$ ). On the other hand, the treatment of $0.1 \mu \mathrm{M}$ calycosin had no significant effects on the apoptosis ability of GMCs treated by Ang II $(p>0.05)$.

Calycosin Can Inhibit the Activation of ERK Signaling
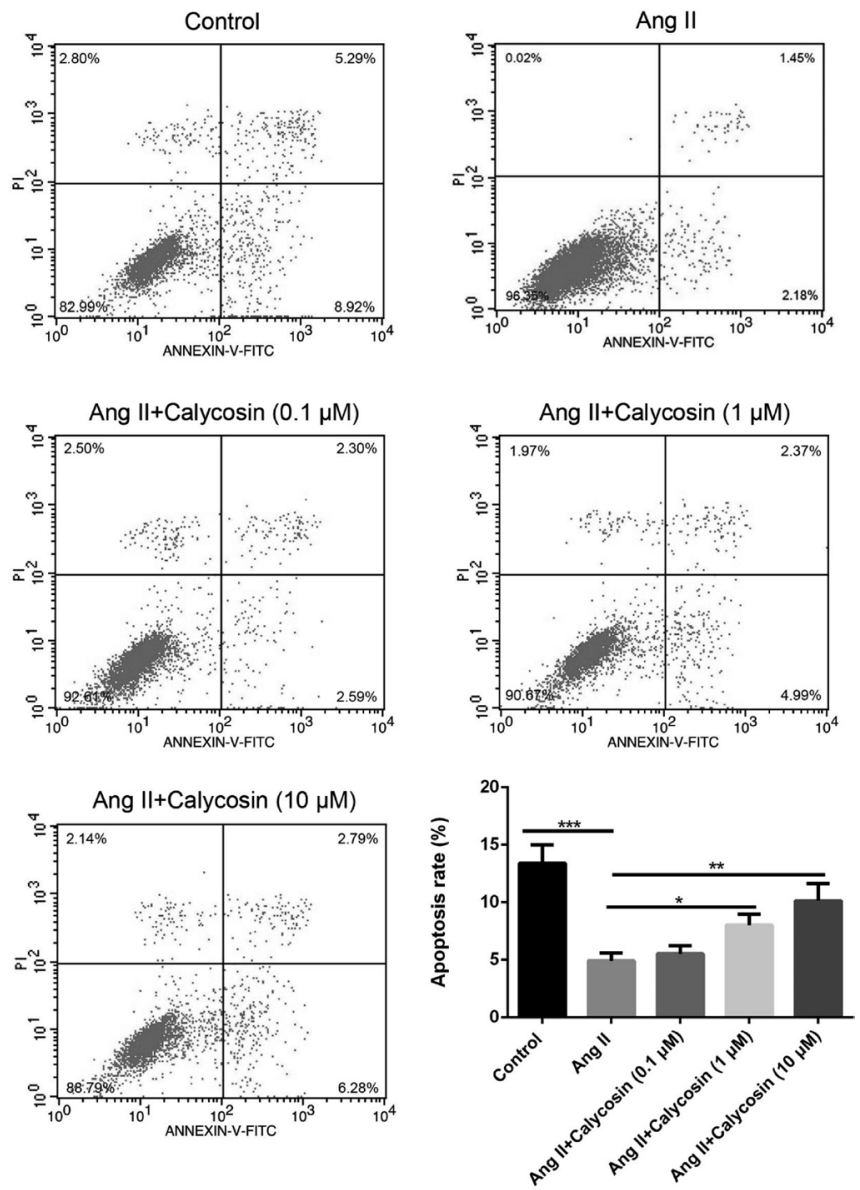

Fig. 4. Effect of Calycosin on the Apoptosis of Glomerular Mesangial Cells Treated by Ang II in Vitro by Flow Cytometry Assay

$$
* p<0.05, * * p<0.01, * * * p<0.001 \text {. }
$$



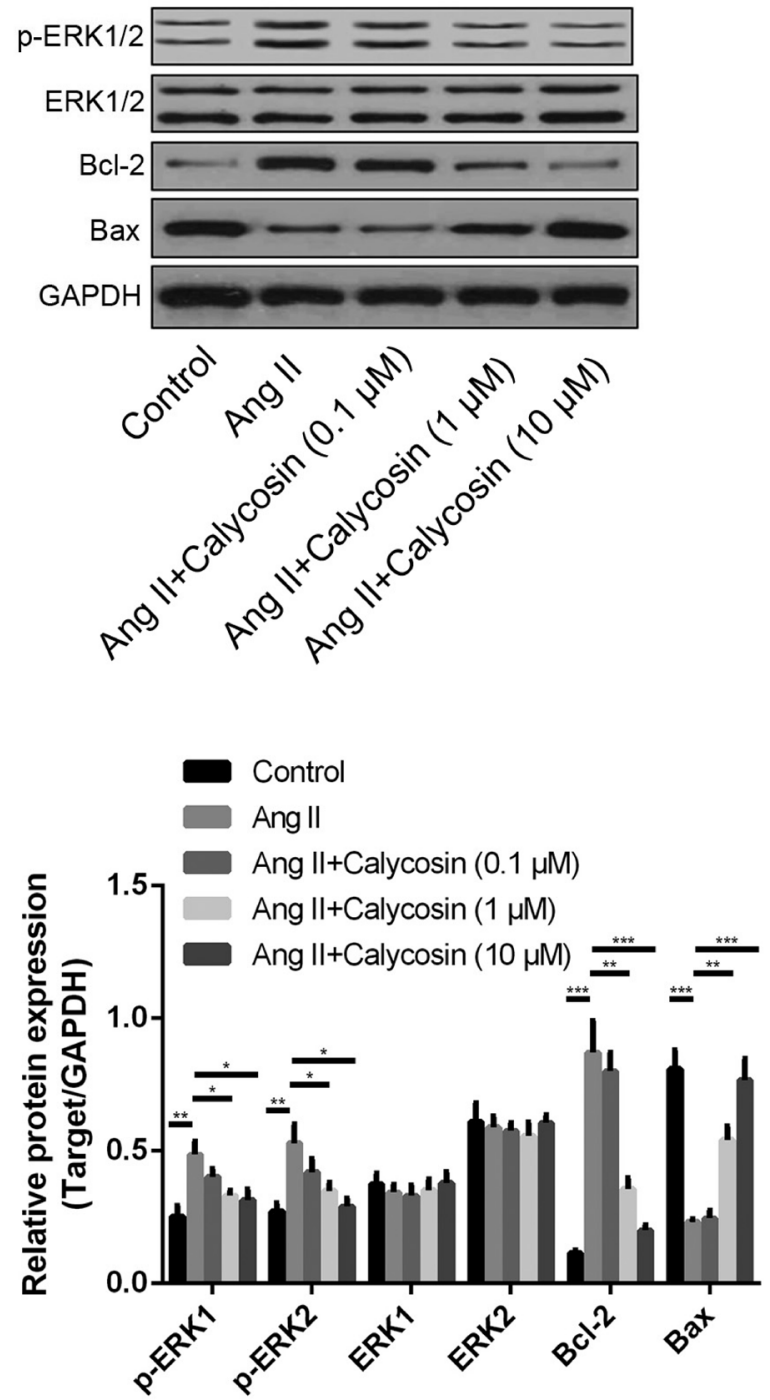

Fig. 5. Effect of Calycosin on the Expression of p-ERK, Bax and Bcl-2 in Glomerular Mesangial Cells Treated by Ang II in Vitro by WB Assay

$* p<0.05, * * p<0.01, * * * p<0.001$.

in GMCs Treated by Ang II in Vitro Finally, the levels of p-ERK, Bcl-2 as well as Bax in GMCs with different treatment at $48 \mathrm{~h}$ were determined by Western blotting (WB) assay. We found that treatment of Ang II markedly increased protein levels of p-ERK and Bcl-2, while on the other hand inhibited the expressions of Bax in glomerular mesangial cells (Fig. 5, $p<0.05$ ); moreover, 1 and $10 \mu \mathrm{M}$ calycosin decreased the levels of p-ERK as well as Bcl-2, while on the other hand increased Bax expression in GMCs treated by Ang II $(p<0.05)$. $0.1 \mu \mathrm{M}$ calycosin did not show significant inhibitory effects on ERK signaling in GMCs treated by Ang II $(p>0.05)$.

Calycosin Can Inhibit the Autophagy of GMCs Induced by Ang II Finally, we examined whether calycosin could affect the autophagy GMCs treated by Ang II using WB and immunocytochemistry methods to examine the expression of the autophagic marker LC-3, Beclin-1, and P62. We found that treatment of Ang II markedly increased the protein levels of LC-3, Beclin-1 expression and decreased P62 expression in GMCs, (Figs. 6, 7, $p<0.001$ ); on the other hand, 1 and $10 \mu \mathrm{M}$ calycosin inhibited the protein expression of LC-3 and Beclin-1 and increased the expression of P62 in GMCs treated

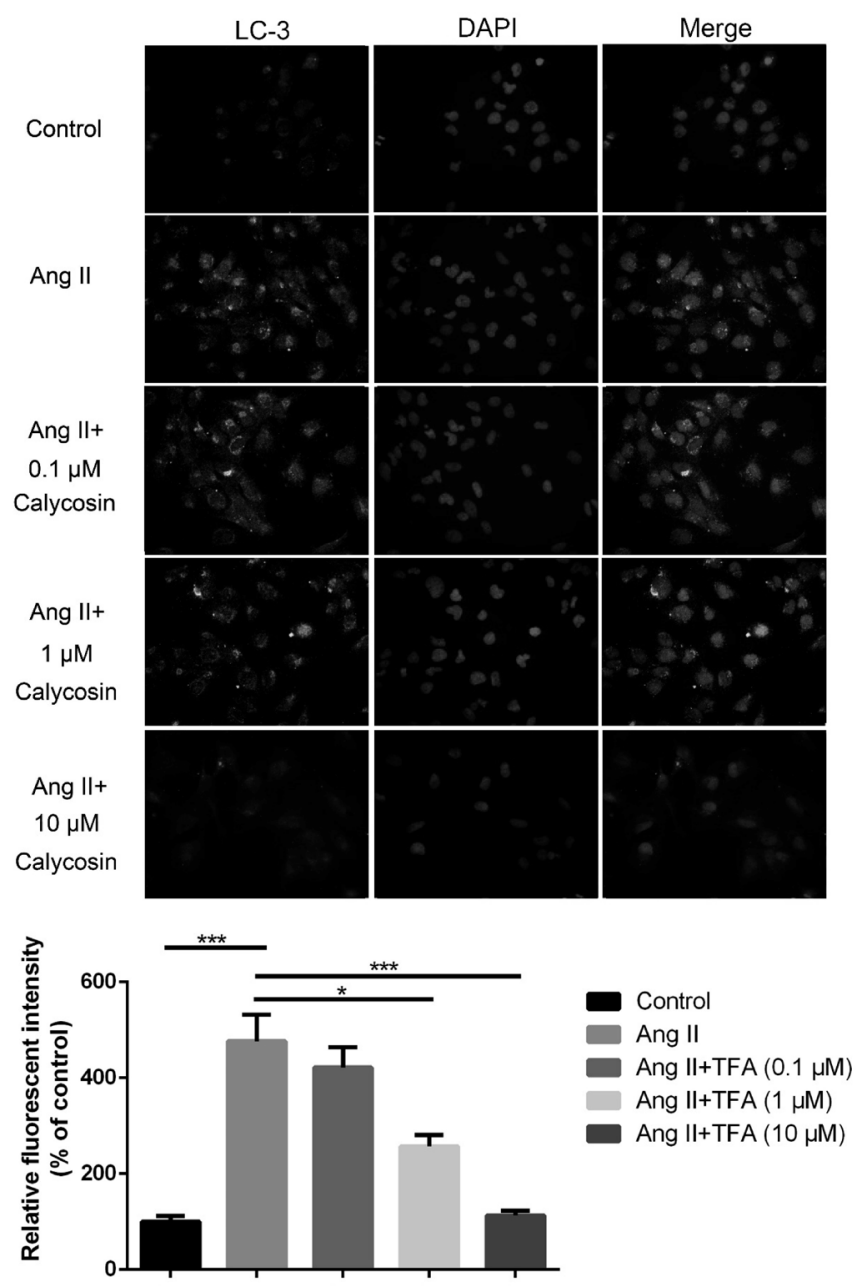

Fig. 6. Effect of Calycosin on the Expression of LC-3 in Glomerular Mesangial Cells Treated by Ang II in Vitro by Immunocytochemistry Assay

$$
* p<0.05, * * p<0.01, * * * p<0.001 .
$$

by Ang II in a dose-dependent manner (Figs. 6, 7, $p<0.05$ ). $0.1 \mu \mathrm{M}$ calycosin did not affect the autophagy of GMCs treated by Ang II $(p>0.05)$.

\section{DISCUSSION}

In this study, the roles of calycosin on the growth, apoptosis, and autophagy of Ang II treated glomerular mesangial cells and discussed the possible mechanism have been explored. We found that calycosin can alleviate Ang II-induced pro-proliferative and anti-apoptotic effects on glomerular mesangial cells, possible via inhibiting the autophagy and suppressing the ERK signaling pathway. The results of the current work suggested that calycosin may be used as an alternative medication for treating chronic kidney disease.

The protective roles of natural compounds in renal diseases have been discussed previously. For example, tectorigenin has been reported to alleviate diabetic nephropathy via regulating the AdipoR1/2 signaling; magnesium lithospermate B was able to exert renal protective roles via suppressing the mitochondrial pathway of apoptosis ${ }^{10)}$; astragaloside IV was shown to inhibit the epithelial-mesenchymal transition of podocytes that induced by glucose through regulating the sirtuin (SIRT)nuclear factor-kappaB (NF- $\kappa \mathrm{B})$ p65 signaling. ${ }^{21)}$ Calycosin has 

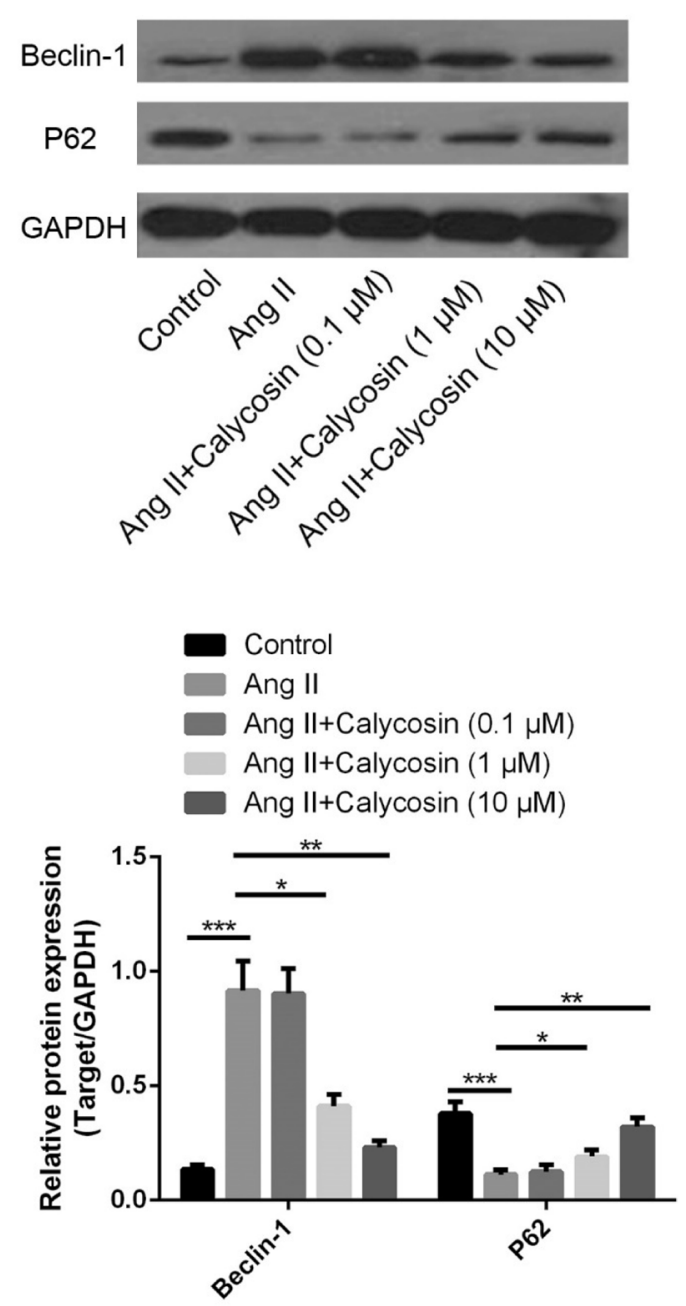

Fig. 7. Effect of Calycosin on the Expression Beclin-1 and P62 in Glomerular Mesangial Cells Treated by Ang II in Vitro WB Assay

$* p<0.05, * * p<0.01, * * * p<0.001$.

been reported as an alternative medication for the treatment of some diseases e.g., cardiovascular diseases, cancer, inflammatory diseases ${ }^{17,22,23)}$; however, it remains unclear whether calycosin could affect the behaviors of Ang II treated glomerular mesangial cells.

In current work, consistent with previous findings, Ang II was shown to significantly increased growth while on the other hand inhibited the apoptosis ability of glomerular mesangial cells, ${ }^{24)}$ which confirmed roles of Ang II in the development of chronic kidney disease. More importantly, while treatment of only calycosin without Ang II did not significantly affect the growth and apoptosis of GMCs, we reported for the first time that 1 and $10 \mu \mathrm{M}$ calycosin can inhibit the growth and promote apoptosis of the GMCs treated by Ang II, suggesting that calycosin can alleviate Ang II induced pro-proliferative and antiapoptotic effects in glomerular mesangial cells.

ERK signaling pathway was known as important signaling that regulates the glomerular mesangial cell growth as well as apoptosis. ${ }^{25-27)}$ Previous studies suggested that the treatment of Ang II can activate the ERK signaling pathway in glomerular mesangial cells. ${ }^{28)}$ Nevertheless, whether calycosin alleviates Ang II-induced over-proliferative and anti-apoptotic effects in glomerular mesangial cells via inhibiting ERK signaling still requires further investigation. In current work, we found that treatment of 1 and $10 \mu \mathrm{M}$ calycosin markedly inhibited the expression of phosphorylated ERK in GMCs treated by Ang II; moreover, calycosin also increased the level of proapoptotic factor Bax, and increase the level of anti-apoptotic factor Bcl-2 (both of them were the downstream proteins and of ERK signaling) in GMCs treated by Ang II. Therefore, our data suggested that calycosin may regulate growth and apoptosis of glomerular mesangial cells, at least partially, through inhibiting the ERK signaling.

Autophagy, also known as "programmed cell death type II," has become an area of focus in recent years. ${ }^{29-31)}$ While in most circumstances, the autophagy of the cells was maintained at low; while on the other hand, when cells were facing undesired conditions (for example, oxidative stress, heat, changes of the microenvironment etc.), the autophagy of the cells may significantly increase and lead to decreased apoptosis of the cells. ${ }^{32,33)}$ Ang II was known to increase the autophagy of GMCs, ${ }^{34,35)}$ and interestingly, in a recent work, calycosin has shown anti-autophagic effects in cerebral ischemic and reperfusion injury models. ${ }^{36)}$ In the present study, Ang II increased the autophagy of GMCs, which was consistent with the previous findings. More importantly, we first reported that 1 and $10 \mu \mathrm{M}$ calycosin could inhibit Ang II induced autophagy of GMCs in a dose-dependent manner. Therefore, we speculated that calycosin may exert its anti-proliferative effects on Ang II treated GMCs via inhibiting the autophagy of the cells. However, the roles of autophagy in renal disease in controversial. some studies have reported that autophagy may play positive roles in renal disease by reducing renal dysfunction. ${ }^{37,38)}$ Therefore, the results of the present study remain to be further investigated by animal and clinical studies.

Current work has limitations. First, further in vivo study as well as the animal study is needed to support our conclusion; second, the roles of other signaling pathways (for example, Akt signaling) during this process should be evaluated; moreover, LC3-I to -II conversion should also be studied in future to confirm the current results.

To sum up, the present work demonstrated that calycosin may alleviate Ang II-induced pro-proliferative and anti-apoptotic effects on glomerular mesangial cells at least partially via decreasing the autophagy and inhibiting the ERK signaling. The results of current work have provided theoretical basis for the potential clinical use of calycosin as an alternative medication in treating chronic kidney disease.

Conflict of Interest The authors declare no conflict of interest.

\section{REFERENCES}

1) Nelson T, Velazquez H, Troiano N, Fretz JA. Early B cell factor 1 (EBF1) regulates glomerular development by controlling mesangial maturation and consequently COX-2 expression. J. Am. Soc. Nephrol., 30, 1559-1572 (2019).

2) Mehta N, Gava AL, Zhang D, Gao B, Krepinsky JC. Follistatin protects against glomerular mesangial cell apoptosis and oxidative stress to ameliorate chronic kidney disease. Antioxid. Redox Signal., 31, 551-571 (2019).

3) Das F, Maity S, Ghosh-Choudhury N, Kasinath BS, Ghosh Choudhury G. Deacetylation of S6 kinase promotes high glucose-induced glomerular mesangial cell hypertrophy and matrix protein accumulation. J. Biol. Chem., 294, 9440-9460 (2019). 
4) Takaki T, Ohno N, Saitoh S, Nagai M, Joh K. Podocyte penetration of the glomerular basement membrane to contact on the mesangial cell at the lesion of mesangial interposition in lupus nephritis: three-dimensional analysis by serial block-face scanning electron microscopy. Clin. Exp. Nephrol., 23, 773-781 (2019).

5) $\mathrm{Hu}$ TY, Li LM, Pan YZ. CTRP3 inhibits high glucose-induced human glomerular mesangial cell dysfunction. J. Cell. Biochem., 120, 5729-5736 (2019).

6) Lu Q, Ma Z, Ding Y, Bedarida T, Chen L, Xie Z, Song P, Zou MH. Circulating miR-103a-3p contributes to angiotensin II-induced renal inflammation and fibrosis via a SNRK/NF-kappaB/p65 regulatory axis. Nat. Commun., 10, 2145 (2019).

7) Colombo R, Santos RA, Rech LS, Tonolli BT, Farina GS, Gerhardt GJL. An educational tool to improve understanding of angiotensin II function and the adrenergic system in renal circulation. $A d v$. Physiol. Educ., 43, 529-533 (2019).

8) Krátký V, Kikerlova S, Huskova Z, Sadowski J, Kolar F, Cervenka L. Enhanced renal vascular responsiveness to angiotensin II and norepinephrine: a unique feature of female rats with congestive heart failure. Kidney Blood Press. Res., 44, 1128-1141 (2019).

9) Gómez GI, Velarde V, Saez JC. Role of a RhoA/ROCK-dependent pathway on renal connexin43 regulation in the angiotensin IIinduced renal damage. Int. J. Mol. Sci., 20, 4408 (2019).

10) Yang S, Ma C, Wu H, Zhang H, Yuan F, Yang G, Yang Q, Jia L, Liang Z, Kang L. Tectorigenin attenuates diabetic nephropathy by improving vascular endothelium dysfunction through activating AdipoR1/2 pathway. Pharmacol. Res., 153, 104678 (2020).

11) Zhang D, Zhuang Y, Pan J, Wang H, Li H, Yu Y, Wang D. Investigation of effects and mechanisms of total flavonoids of Astragalus and calycosin on human erythroleukemia cells. Oxid. Med. Cell. Longev., 2012, 209843 (2012).

12) Wang D, Zhuang Y, Tian Y, Thomas GN, Ying M, Tomlinson B. Study of the effects of total flavonoids of Astragalus on atherosclerosis formation and potential mechanisms. Oxid. Med. Cell. Longev., 2012, 282383 (2012).

13) Tian W, Wang ZW, Yuan BM, Bao YG. Calycosin induces apoptosis in osteosarcoma cell line via ERbetamediated PI3K/Akt signaling pathways. Mol. Med. Rep., 21, 2349-2356 (2020).

14) Liu X, Sun X, Deng X, Lv X, Wang J. Calycosin enhances the bactericidal efficacy of polymyxin B by inhibiting MCR-1 in vitro. $J$. Appl. Microbiol., 129, 532-540 (2020).

15) Wu ML, Lin YP, Wei YL, Du HJ, Ying XQ, Tan WZ, Tang BE. Calycosin influences the metabolism of five probe drugs in rats. Drug Des. Devel. Ther., 14, 429-434 (2020).

16) Zhai J, Tao L, Zhang S, Gao H, Zhang Y, Sun J, Song Y, Qu X. Calycosin ameliorates doxorubicin-induced cardiotoxicity by suppressing oxidative stress and inflammation via the sirtuin 1-NOD-like receptor protein 3 pathway. Phytother. Res., 34, 649-659 (2020).

17) El-Kott AF, Al-Kahtani MA, Shati AA. Calycosin induces apoptosis in adenocarcinoma HT29 cells by inducing cytotoxic autophagy mediated by SIRT1/AMPK-induced inhibition of Akt/mTOR. Clin. Exp. Pharmacol. Physiol., 46, 944-954 (2019).

18) Elsherbiny NM, Said E, Atef H, Zaitone SA. Renoprotective effect of calycosin in high fat diet-fed/STZ injected rats: Effect on IL-33/ST2 signaling, oxidative stress and fibrosis suppression. Chem. Biol. Interact., 315, 108897 (2020).

19) Liu J, Deng T, Wang Y, Zhang M, Zhu G, Fang H, Wang J. Calycosin inhibits intestinal fibrosis on CCD-18Co cells via modulating transforming growth factor-beta/Smad signaling pathway. Pharmacology, 104, 81-89 (2019).

20) Zhang YY, Tan RZ, Zhang XQ, Yu Y, Yu C. Calycosin ameliorates diabetes-induced renal inflammation via the NF-kappaB pathway in vitro and in vivo. Med. Sci. Monit., 25, 1671-1678 (2019).

21) Wang X, Gao Y, Tian N, Wang T, Shi Y, Xu J, Wu B. Astragaloside IV inhibits glucose-induced epithelial-mesenchymal transition of podocytes through autophagy enhancement via the SIRT-NF-
kappaB p65 axis. Sci. Rep., 9, 323 (2019).

22) Zhang Z, Auyeung KK, Sze SC, Zhang S, Yung KK, Ko JK. The dual roles of calycosin in growth inhibition and metastatic progression during pancreatic cancer development: A "TGF-beta paradox". Phytomedicine, 68, 153177 (2020).

23) Yan X, Yu A, Zheng H, Wang S, He Y, Wang L. Calycosin-7-Obeta-D-glucoside attenuates OGD/R-induced damage by preventing oxidative stress and neuronal apoptosis via the SIRT1/FOXO1/PGC1alpha pathway in HT22 cells. Neural Plast., 2019, 8798069 (2019).

24) Zhang N, Ji Z. Effects of caveolin-1 and P-ERK1/2 on Ang IIinduced glomerular mesangial cell proliferation. Ren. Fail., 35, 971-977 (2013).

25) Sun Y, Shi X, Peng X, Li Y, Ma H, Li D, Cao X. MicroRNA-181a exerts anti-inflammatory effects via inhibition of the ERK pathway in mice with intervertebral disc degeneration. J. Cell. Physiol., 235, 2676-2686 (2020).

26) Kong J, Li L, Lu Z, Song J, Yan J, Yang J, Gu Z, Da Z. MicroRNA-155 suppresses mesangial cell proliferation and TGF-betal production via inhibiting CXCR5-ERK signaling pathway in lupus nephritis. Inflammation, 42, 255-263 (2019).

27) $\mathrm{Xu} \mathrm{JL}$, Gan XX, Ni J, Shao DC, Shen Y, Miao NJ, Xu D, Zhou L, Zhang W, Lu LM. SND p102 promotes extracellular matrix accumulation and cell proliferation in rat glomerular mesangial cells via the AT1R/ERK/Smad3 pathway. Acta Pharmacol. Sin., 39, 1513-1521 (2018).

28) Gorin Y, Ricono JM, Wagner B, Kim NH, Bhandari B, Choudhury GG, Abboud HE. Angiotensin II-induced ERK1/ERK2 activation and protein synthesis are redox-dependent in glomerular mesangial cells. Biochem. J., 381, 231-239 (2004).

29) Li W, Ren Y, Meng T, Yang W, Zhang W. miR-129-5p attenuates hypoxia-induced apoptosis in rat $\mathrm{H} 9 \mathrm{c} 2$ cardiomyocytes by activating autophagy. J. Gene Med., 22, 3200 (2020).

30) Barnhill LM, Khuansuwan S, Juarez D, Murata H, Araujo JA, Bronstein JM. Diesel exhaust extract exposure induces neuronal toxicity by disrupting autophagy. Toxicol. Sci., 176, 193-202 (2020).

31) Nian JB, Zeng M, Zheng J, Zeng LY, Fu Z, Huang QJ, Wei X. Epithelial cells expressed IL-33 to promote degranulation of mast cells through inhibition on ST2/PI3K/mTOR-mediated autophagy in allergic rhinitis. Cell Cycle, 19, 1132-1142 (2020).

32) Hao R, Li F, Song X, Tan X, Sun-Waterhouse D, Li D. Caffeic acid phenethyl ester against cadmium induced toxicity mediated by CircRNA modulates autophagy in HepG2 cells. Ecotoxicol. Environ. Saf., 197, 110610 (2020).

33) Zhao X, Shi Y, Zhang D, Tong X, Sun Y, Xue X, Fu J. Autophagy inducer activates Nrf2-ARE pathway to attenuate aberrant alveolarization in neonatal rats with bronchopulmonary dysplasia. Life Sci., 252, 117662 (2020).

34) Yang S, Sun D, Wang L, Wang X, Shi M, Jiang X, Gao X. The role of STAT3/mTOR-regulated autophagy in angiotensin II-induced senescence of human glomerular mesangial cells. Cell. Signal., 53, 327-338 (2019).

35) Shen Q, Bi X, Ling L, Ding W. 1,25-Dihydroxyvitamin D3 attenuates angiotensin II-induced renal injury by inhibiting mitochondrial dysfunction and autophagy. Cell. Physiol. Biochem., 51, 1751-1762 (2018).

36) Wang Y, Ren Q, Zhang X, Lu H, Chen J. Neuroprotective mechanisms of calycosin against focal cerebral ischemia and reperfusion injury in rats. Cell. Physiol. Biochem., 45, 537-546 (2018).

37) Ma Z, Li L, Livingston MJ, Zhang D, Mi Q, Zhang M, Ding HF, Huo Y, Mei C, Dong Z. p53/microRNA-214/ULK1 axis impairs renal tubular autophagy in diabetic kidney disease. J. Clin. Invest., 130, 5011-5026 (2020).

38) Zhang P, Fang J, Zhang J, Ding S, Gan D. Curcumin inhibited podocyte cell apoptosis and accelerated cell autophagy in diabetic nephropathy via regulating Beclin1/UVRAG/Bcl2. Diabetes Metab. Syndr. Obes., 13, 641-652 (2020). 